\title{
Chronic endometritis and the state of the receptor apparatus in women with reproductive losses
}

\begin{abstract}
The purpose of this study was to determine the expression of lymphocytes and content of estrogen receptors (ER) and progesterone (PR) in the stroma and epithelium of the endometrium in women with histologically confirmed endometritis. Surveyed 56 women with infertility and miscarriage, in which the immune histological method was used to determine ER and PR in stroma and epithelium of the endometrium and expression of lymphocytes. It is revealed that the inflammatory reaction in the endometrium was expressed mainly in the lymphocytic infiltration and was predominantly $\mathrm{CD} 3, \mathrm{CD} 4, \mathrm{CD} 8$ lymphocytes. The affected endometrial receptivity has been broken: the middle phase of proliferation in the stroma and in the endometrium was dominated by progesterone receptors. The ratio of ER and PR was $<1$, indicating a lack of maturity and readiness of the endometrium to its secretory transformation and the adoption of a blastocyst. Despite the low expression of ER and focal hyperplasia of the basal layer occurred $53,10 \%$ of women. The incidence of hyperplasia correlated with the expression of $\mathrm{CD} 3$ and $\mathrm{CD} 68$ cells.
\end{abstract}

Keywords: infertility, habitual miscarriage, chronic endometritis, receptors to estrogens, progesterone receptors, immunohistochemistry, lymphocytes CD3, CD4, CD8

\author{
Volume 4 Issue 2 - 2018 \\ Voznesenskaya Nadezda, Kozyreva Elena, \\ Gafurova Diana, Galiullina Leysan \\ Ulyanovsk state University, Ulyanovsk, Russia
}

Correspondence: Voznesenskaya Nadezda, Ulyanovsk state University, Ulyanovsk, Russia, Email vnadezda@list.ru

Received: February 25, 2018| Published: April 26, 2018

\section{Introduction}

Chronic endometritis (CE) is a clinical and morphological syndrome, in which as a result of persistent damage to the endometrium by an infectious agent, multiple secondary morphofunctional changes occur that violate the cyclic transformation and receptivity of the uterine mucosa. ${ }^{1,2}$

According to the decision of the XVIII world Congress of gynecologists and obstetricians (FIGO, 2006), ${ }^{3,4}$ the diagnosis of chronic endometritis should put all women who have had nondeveloping pregnancy, not performing more tests. In addition to $\mathrm{CE}$, clinicians often face this contingent of patients with the situation of "escape" of the endometrium from under normal hormonal influences and desynchronization of ovarian and endometrial cycles, insufficient growth of endometrium, especially in ovarian stimulation programs in patients with infertility. 5,6 The receptivity of the endometrium is a complex of structural and functional characteristics of the endometrium with clear time constants that determine the ability of the endometrium to provide embryo implantation. Currently, the most widely used in clinical practice to determine the receptor apparatus is immunohistochemical verification of estrogen receptor (ER) and progesterone receptor (PR) in endometrial tissue. ${ }^{7,8}$ The amount of estrogen and progesterone receptors varies throughout the normal menstrual cycle. ${ }^{9}$ Depending on the stage and phase of the menstrual cycle, these or those receptors prevail in the epithelium of the glands or stromal component.

\section{Objective}

To study the expression of lymphocytes and estrogen and progesterone receptors in stroma and endometrial epithelium in women with CE. A comprehensive survey of 56 women with reproductive problems: 36 women had a history of habitual pregnancy loss and 20 had infertility.

Ultrasonography (USG) of the pelvic organs was performed on 20-22 day of the menstrual cycle. The indicators of endometrium thickness and structure were evaluated. The uniform structure and its correspondence to the day of menstrual cycle, the thickness of endometrium were considered as normal parameters not less than $8 \mathrm{~mm}$.

In all examined in histological examination of the endometrium, $\mathrm{CE}$ was diagnosed. For histological examination, the endometrium was obtained by a pipel biopsy, which was performed according to the standard method on the 7-11 day of the menstrual cycle.

Immunohistochemical (IHC) examination of endometrial tissue it was carried out using the method of peroxidase technique on paraffin sections 3-4 microns thick. The system has been used for the detection UltraVision Quanto (DAKO, Denmark) and monoclonal mouse antibodies firm "DAKO": estrogen receptor (clone 1D5), progesterone (clone PgR 636), for receptors of lymphocytes - CD3 (clone SP-7); CD4 (clone 4B12); CD8 (clone), CD138 (clone).

To determine the expression level of estrogen and progesterone receptors, a semi-quantitative system "Allred Scoring Guidline" was used to estimate the percentage of colored cells (proportion score, PS) and the intensity of cell coloring (intensity score, IS), which were then summed up: TS (total score) $=\mathrm{PS}+$ is. The percentage of stained cells was assessed in points: $0-0 \%$ cells, $1-0,1-1 \%$ of cells, $2-10 \%$ of cells, $3-11-33 \%$ of cells, $4-34-66 \%$ of cells, $5-67-100 \%$ of cells. The intensity of staining was assessed in points: 0-no staining, 1-weak staining, 2-moderate, 3-marked coloring. Thus, the result of Allred Score ranged from 0 to 8 points. 
Viewing and photographing of micro-products was carried out on the microscope AxioLab ("Carl Zeiss Jena", Germany) using a digital camera ("Carl Zeiss Jena", Germany). Quantitative analysis of the results of immunohistochemical reactions was carried out under the lens magnification of $20 \times, 40 \times$. Statistical data analysis was carried out on a personal computer using the software package "Statistica" version 7. Used non-parametric methods of statistical analysis. When comparing the performance of different groups used the student's t-test, Mann-Whitney, Wald-Wolfowitz. Significant differences between groups were considered at the significance level of $p<0.05$ Research result.

In patients with ultrasound, the echo structure of the endometrium was changed in $69 \%$ of cases. In the structure of the ultrasonic signs of CE prevailed: heterogeneous echo - structure of the endometrium, its uneven contour and increased echogenicity. Less frequently observed hyperechogenic inclusions in the basal layer of the endometrium, gaseous bubbles in the uterine cavity and its extension.

The bacteriological study of aspirates from the maternal cavity showed no microflora growth in $81 \%$ of infertile women and $79 \%$ of the usual pregnancy loss $(\mathrm{p}>0.05)$. Structure of detected pathogens Staphylococcus epidermidis; Enterococcus faecalis; Staphylococcus sapphyticus; Staphylococcus aureus.

Morphological criteria for the diagnosis of "chronic endometritis" were: inflammatory infiltrates, consisting mainly of lymphocytes, neutrophilic leukocytes, located more often around the glands and blood vessels, less diffuse; the presence of plasma cells $;{ }^{10}$ endometrial stroma alterations, including fibrosis; sclerotic changes in spiral arteries ${ }^{11}$ deformation of the glands; focal hyperplasia of the basal layer. ${ }^{12,13}$

In immunohistochemical study of plasma cells, CD138, 4,5\% of the examined patients were identified. The presence of lymphohistiocytic only infiltration in the stroma of endometrium without plasma cells was evaluated, the picture is incomplete as CE. Focal endometritis occurred in $13.2 \%$, and diffuse -in $86,8 \%$ of women.

In women with chronic diffuse endometritis in the middle of the proliferation phase, the expression of ER in the stroma made up $181,11 \pm 50,0$, in the epithelium $-198,89 \pm 28,72$, while expression of PR was higher in the stroma and in the epithelium, accounting for $220,0 \pm 40.0$ or $237,78 \pm 28,72$ respectively. To ensure the maturity of the endometrium and its readiness for secretory transformation and blastocyst administration, it is important not only the actual number of steroid receptors, but also their ratio. Determining the ratio of ER to PR, the following results were obtained: 0.89 in stromal and 0.86 in glandular components of the endometrium. Lymphocytic reaction in diffuse and focal endometritis did not differ in the qualitative and quantitative composition of lymphocytes and was represented by CD2-35.4\%, CD3-81.8\%, CD4-54.5\%, CD8-77.3\%, CD68-40.9\%. Despite the decreased expression of RE focal hyperplasia, there were $53.10 \%$ of women. The incidence of hyperplasia correlated with the expression of CD3 and CD68 cells (Tables 1-3).

Table I The incidence of focal hyperplasia in the expression of different types of lymphocytes (T-tests)

\begin{tabular}{lllllllll}
\hline & Mean & Mean & t-value & $\mathbf{p}$ & Std.dev. & Std.dev. & F-ratio & p \\
\hline CD2 & 0,61535 & 0,55555 & 0,14835 & 0,88355 & $1,04,390$ & 0,72648 & $2,06,478$ & 0,30964 \\
CD3 & $1,76,923$ & 0,88888 & $2,32,903$ & $0,03045^{*}$ & $1,01,273$ & 0,60092 & $2,84,024$ & 0,14626 \\
CD4 & $1,23,076$ & 0,66666 & $1,23,176$ & 0,23232 & $1,16,575$ & 0,86602 & $1,81,197$ & 0,40579 \\
CD8 & $1,69,230$ & $1,11,111$ & $1,28,798$ & 0,21245 & $1,18,213$ & 0,78173 & $2,28,671$ & 0,24684 \\
CD68 & $1,30,769$ & 0,11111 & $2,93,674$ & $0,00815^{*}$ & $1,18,213$ & 0,33333 & $1,25,769$ & $0,00131^{*}$ \\
CD138 & 0,07692 & 0,000000 & 0,82572 & 0,41870 & 0,27735 & 0,00000 & 0,00000 & $1,00,000$
\end{tabular}

*, Indicators are statistically significant at $\mathrm{p}<0.05$

Table 2 The incidence of focal hyperplasia in the expression of different types of lymphocytes (Mann-Whitney U Test)

\begin{tabular}{lllllllll}
\hline & Rank Sum & Rank Sum & $\mathbf{U}$ & $\mathbf{Z}$ & p-level & $\mathbf{Z}$ & p-level & $\mathbf{2}^{*} \mathbf{1}$ sided \\
\hline CD2 & $14,60,000$ & $10,70,000$ & $55,00,000$ & 0,200334 & 0,841219 & 0,233646 & 0,815260 & 0,844582 \\
CD3 & $18,10,000$ & $7,20,000$ & $27,00,000$ & $20,70,120$ & 0,038442 & $21,66,455$ & $0,030277^{*}$ & 0,036416 \\
CD4 & $16,55,000$ & $8,75,000$ & $42,50,000$ & $10,35,060$ & 0,300642 & $11,02,840$ & 0,270098 & 0,292051 \\
CD8 & $16,80,000$ & $8,50,000$ & $40,00,000$ & $12,02,005$ & 0,229362 & $12,45,299$ & 0,213023 & 0,234852 \\
CD68 & $18,25,000$ & $7,05,000$ & $25,50,000$ & $21,70,287$ & 0,029986 & $24,54,152$ & $0,014122^{*}$ & 0,025202 \\
CD138 & $15,40,000$ & $9,90,000$ & $54,00,000$ & 0,267112 & 0,789383 & 0,739600 & 0,459543 & 0,793760 \\
\hline
\end{tabular}

*, Indicators are statistically significant at $\mathrm{p}<0.05$ 
Table 3 The incidence of focal hyperplasia in the expression of different types of lymphocytes (Wald-Wolfowitz Runs Test)

\begin{tabular}{llllllll} 
& & Mean & Mean & $\mathbf{Z}$ & p-level & $\mathbf{Z}$ adjstd & p-level \\
\cline { 2 - 6 } CD2 & 0,615385 & 0,555556 & $1,52,253$ & 0,127878 & $12,96,207$ & 0,194905 \\
CD3 & $17,69,231$ & 0,888889 & $-1,19,333$ & 0,232740 & 0,967012 & 0,333539 \\
CD4 & $12,30,769$ & 0,666667 & $-0,74069$ & 0,458882 & 0,514368 & 0,606995 \\
CD8 & $16,92,308$ & $11,11,111$ & $-0,28805$ & 0,773312 & 0,061724 & 0,950783 \\
CD68 & $13,07,692$ & 0,111111 & $-2,55,126$ & $0,010734^{*}$ & $23,24,943$ & $0,020076^{*}$ \\
CD138 & 0,076923 & 0,000000 & 0,61724 & 0,537076 & 0,390920 & 0,695857 \\
\hline
\end{tabular}

*, Indicators are statistically significant at $p<0.05$

Thus, the inflammatory reaction in endometrium in women with reproductive problems was mainly expressed in lymphocytic infiltration and was mainly represented by CD3, CD4, CD8 lymphocytes. The affected endometrial receptivity has been broken: the middle phase of proliferation in the stroma and in the endometrium was dominated by progesterone receptors. The ratio of ER and PR was $<1$, which testified to the lack of maturity of the endometrium and its readiness for secretory transformation and blastocyst acceptance. Despite the decreased ER expression, focal hyperplasia of the basal layer occurred in $53.10 \%$ of women. The incidence of hyperplasia correlated with the expression of CD3 and CD68 cells.

\section{Acknowledgements}

None.

\section{Conflict of interest}

The authors declare there is no conflict of interest.

\section{References}

1. Dyuzheva EV. Hormonal preparation of the endometrium in patients with ineffective attempts at IVF in history. Dis Kand Honey Sciences; 2010.

2. Kamenetsky BA. The Use of ultrasound scanning of endometrium in assisted reproduction programs. Problems of Reproduction; 2001.

3. Sukhikh GT. Shurshalina AV. Chronic endometritis. GEOTAR-Media; 2013

4. Radzinsky VE, Gordeev AN. Topical issues of modern obstetrics (based on the materials of the XVIII Figo Congress, 2006). Obstetrics and gynecology. 2007;6:83-85.
5. Miwa I, Tamura H, Takasaki A, et al. Pathophysiologic features of «thin» endometrium. Fertil Steril. 2009;91(4):998-1004.

6. Boyarsky KY, Gaidukov SN, Polchenko N. A modern take on the problem of receptivity defects and thin endometrium in art programs: a review of the literature. Problems of reproduction. 2013;4:51-60.

7. Coutifaris C, Myers ER, Guzick DS. Histological dating of timed endometrial biopsy tissue is not related to fertility status. Fertility Sterility. 2004;82(5):1264-1272.

8. Ellinida VN, Anikeeva N. Maximov. Practical immunohistochemistry (methodological recommendations). SPb: vterm of EMERCOM of Russia; 2002:36.

9. Beato MJ. Klug Steroid hormone receptors: an update. Human Reproduction. 2000;6(3):225-236.

10. Kitaya K, Yasuo T. Inter-observer and intra-observer variability in immunohistochemical detection of endometrial stromal plasmacytes in chronic endometritis. Ехю Ther Med. 2013;5(2):485-488.

11. McQueen DB, Perfetto CO, Hazard FK, et al. Pregnancy outcomes in women with chronic endometritis and recurrent pregnancy loss. Fertil Steril. 2015;104(4):927-931.

12. Devroey P, Bourgain C, Macklon NS. et al. Reproductive biology and IVF: ovarian stimulation and endometrial receptivity. Trends Endocrinology Metabolism. 2004;15(2):84-90.

13. Chen SL, Wu FR, Luo C, et al. Combined analysis of endometrial thickness and pattern in predicting outcome of in vitro fertilization and embryo transfer: a retrospective cohort study. Reprod Biol Endocrinol. 2010;8:30. 